\title{
CAN WE PREDICT THE RESPONSE TO GALANTAMINE IN ALZHEIMER'S DISEASE PATIENTS?
}

\author{
Carmen Gavan", Cristina Tiu ${ }^{1,2}$, Ovidiu Alexandru Bajenaru ${ }^{1,2}$ \\ 1 "Carol Davila" University of Medicine and Pharmacy, Bucharest \\ ${ }^{2}$ University Hospital, Bucharest
}

\begin{abstract}
Although considerate progress was obtained by adding imagistic and laboratory test, clinical examination remains the core of the diagnosis for Alzheimer's disease. After 20 years of experience with anticholinergic drugs we know that only one third of the patients respond to the treatment; the treatment is more efficient in the initial stages of the neurocognitive decline. It is important to evaluate the efficacy of drugs as soon as possible. We examined patients with mild and moderate Alzheimer's disease referred through our outpatient clinic. They fulfilled the NINCDS-ADRDA criteria for Alzheimer's disease and they scored at baseline 21 to 26 points on MMSE. The patients were treated with Galantamine, $8 \mathrm{mg} /$ day for the first month and then $16 \mathrm{mg} / \mathrm{day}$. We evaluated the patients after 2 and 6 months of treatment; we performed MMSE and ADAS-Cog in order to see if a more complex evaluation would be helpful. We considered that the treatment was efficient if MMSE score did not decrease after 6 months of treatment. We also evaluated clinical disability using DAD (Disability Assessment for Dementia Scale) and the difficulty of living with a patient with dementia using CBS (Caregiver Burden Scale). The examination using MMSE and ADAS-Cog after two months of treatment does not have a predictable value. ADAS-Cog examination can be useful for patients that have a stable MMSE score after six months of treatment. Although the evolution of ADAS-Cog and MMSE scores were significantly positive, clinical disability of the patients progressed, rising concerns for their caregivers.
\end{abstract}

Keywords: Alzheimer`s disease, MMSE, ADAS-Cog, DAD, CBS, galantamine

\section{BACKGROUND}

Neurocognitive deterioration affects $10 \%$ of the population aged 65 or more, and about one third of the population aged 85 or more in Europe and in North America. (1) The most frequent type of dementia is Alzheimer's disease (AD). The diagnosis and the classification of the severity of dementia is based upon the Mini Mental State Examination, a test which was elaborated by Folstein in 1975. The presence of memory impairment was compulsory; dementia has many other causes either due to degenerative diseases, or as a complication of other diseases (i.e. fronto-temporal degeneration, dementia secondary to Parkinson disease, Diffuse Lewy Body disease, vascular dementia, or dementia associated with infections, metabolic disorders, trauma, toxic substances a.s.o). In these cases, the first cognitive deficits manifesting in clinical evolution are most often not the memory impairment, but executive, visuospatial and social cognition dysfunction. There is recent evidence that even Alzheimer's disease may manifest when the first clinical signs of dementia appear, not by memory impairment but by other cognitive dysfunctions (e.g. language); this is the background of a particular form of clinical picture of Alzheimer's disease defined as "atypical AD”. (2)

As general population gets older, the incidence of cognitive impairment rises. If at the end of the last century, searching for cognitive dysfunction in the adult population seemed to be rather a caprice than a necessity, nowadays cognitive evaluation is 
mandatory not only in people suspected of AD onset, but also in other diseases, like cerebrovascular disorders, or, in diseases affecting younger adults, such as multiple sclerosis. Facing a person complaining of symptoms suggestive for cognitive dysfunction, the physician must rule out other possible causes (vascular, metabolic, infectious, tumoral) and decide if it is a neurodegenerative disorder. In dementia due to a neurodegenerative process, the therapeutic benefits of the available drugs are seen in the early stages of the disease. This makes early diagnosis of paramount importance for the planning of the resources available in the social care system. Although a significant progress has been made in understanding the pathophysiologic mechanism behind cognitive impairment, the investigations proved to have a good sensibility and specificity for $\mathrm{AD}$ diagnosis available today, cannot be used for general population screening; they are far too complex, expensive, invasive and time consuming to be used in the primary health care system (PET scan with radiotracer for beta-amyloid, genetic testing for APOE- $\varepsilon-4$, testing for beta-amyloid and tau protein in the cerebrospinal fluid).

The diagnosis and grading of the cognitive impairment is though, mainly clinical, based on the neurocognitive tests. In order to be used in clinical practice, tests should be performed in a reasonable amount of time, have high specificity and sensibility. They should be able to cover the six domains of interest- attention/working memory, learning and delayed recall, speech performance, visual construction, executive function and abstract thinking.

MMSE - developed by Folstein and McHugh in 1975 is the most widely used test for detection of cognitive impairment. It takes 10 minutes to be performed and the specificity and the sensibility are increased with advancing age and educational status of the patient. It is thus still suboptimal for the target population (3). A score of 20 to 24 suggests mild dementia, 13 to 20 suggests moderate dementia, and less than 12 indicates severe dementia. The test does not take into account the influence of the anxiety, on the interviewed patient's performance and the opinion of the caregiver regarding the performance of the patient.

MoCA (Montreal Cognitive Assessment ) created in 1996 by Ziad Nasreddine, is a test conceived for the diagnosis of the mild cognitive impairment, with sensibility and specificity higher than MMSE; it still has the pitfalls of the MMSE (4) (it does not take into account the opinion of the caregiver and the anxiety of the performance of the patient).
Clock drawing test - it is easy to administrate and is testing the executive function which is affected earlier than memory in certain types of cognitive dysfunction. (5) It has similar sensibility and specificity.

A specific test for the patients with mild to moderate AD was designed in 1984 - Alzheimer's Disease Assessment Scale-cognitive subscale with a new version with 13 items. ADAS-cog was developed in 1984 mainly for clinical research, and has a duration of 30 minutes (6). It contains now 13 items - words recall, naming objects and fingers, following commands, ideational praxis, constructional praxis, orientation, word recognition test, remembering test instruction and three additional subjective scales containing assessment of spoken language ability, word finding difficulty and comprehension. Higher scores indicates a more severe condition; a score higher than 17 points is considered diagnostic for a mild form of dementia and a score higher than 70 points reflects a severe condition.

There are new tests for the assessment of the patient's mood and for the behavioral disorder associated with the neurocognitive impairment. The best example is NPI (Neuropsychiatric Inventory), developed by de Cummings (1994). (7) It uses scales to quantify parameters such as frequency, severity and impact of behavioral disorder associated with neurocognitive impairment on the caregiver. The reference point (before cognitive impairment) to which the caregiver compares the current status of the patient is arbitrary.

DAD (Disability for Dementia Assessment Scale) evaluates the functional disability; it includes 40 items: 17 related to basic self-care and 23 to instrumental activities of daily living. It has demonstrated a high degree of internal consistency and excellent inter-rater and test-retest reliability. In addition, it was found not to have gender bias. This instrument may help clinicians and caregivers of the population with Alzheimer's disease to make decisions regarding the choice of suitable interventions. (8)

Caregiver Burden Scale - contains 22 questions which evaluates the perception of the caregiver regarding the difficulty of living with a person with cognitive impairment (9). This scale was developed on the knowledge that excessive burden on the caregiver results in their increased morbidity, rising the costs of the health care system. Early planning is mandatory in the management of the patient with dementia. (10) 
Clinical Dementia Rating Scale (1982) - assesses the cognitive and functional performance of the patient on the basis of a structural interview with the patient and the caregiver with emphasis on: personal care, daily living, social life, daily finance management, hobbies. (11) The sensibility of CDRS is superior for mild cognitive impairment. $(12,13)$

For the moment there are four drugs approved by FDA and EMA for the treatment of dementia: Donepezil (1996), Rivastigmine (1997), Galantamine (2001) and Memantine (2002). The benefit of these drugs is now considered limited for the treatment of the mild and moderate forms of dementia (14); the improvement of the memory functions is for a short period and sometimes is not seen at all. $(15,16)$ About one third of the patients do not respond to one of these drugs and this means that we all lose time to treat (17).

\section{MATERIAL AND METHODS}

We examined 11 patients, 6 women and 5 men, with a mean age of 65 (57 to 73 years old) with Alzheimer's Disease (AD) treated with Galantamine, a competitive, reversible inhibitor of acetylcholinesterase; galantamine hydrobromide is also an allosteric modulator of the nicotinic receptors of acetylcholine. The patients were referred through the outpatient clinic of our hospital after performing a brain CT scan, MMSE and blood analysis. The patients were diagnosed with mild and moderate forms of dementia, according to NINCDS-ADRDA criteria, scoring on MMSE between 21 to 26 points and did not have a previous treatment for neurocognitive impairment. The patients were selected from a larger group, referred for memory complaints. Patients with previous strokes, or silent strokes identified on brain CT/ MRI were excluded. The patients received Galantamine $8 \mathrm{mg} /$ day for 28 days and $16 \mathrm{mg}$ /day for another 140 days. We considered that the treatment was efficient if the MMSE score did not fall bellow the baseline score.

\section{RESULTS}

The initial group included 11 patients (6 women and 5 men) but only 8 ( 4 women and 4 men) completed the trial; 2 patients reported nausea as a side effect of the drug and one withdrew consent. The age of the patients was between 57 and 73 , with a mean age of 65 (64.875). Three of them were single, the caregiver being a sister/brother or the chil- dren. The level of education was between 8 years to 16 years, with a mean of 13 years. Most of them (7) were hypertensive and had dyslipidemia; one patient had diabetes mellitus type 2 and two patients were treated for depression. The demographic characteristics of the group are presented in Table 1.

After two months of treatment MMSE score did not decrease for any of the patients and only one patient's ADAS-cog score was increased compared with the initial examination. At six months, MMSE score of two patients decreased and ADAS-cog score was raised, one remained the same although the ADAS-cog score decreased, and five showed an improvement of MMSE score and a concomitant decrease of ADAS-cog. The median scores of MMSE raised from 22.625 at baseline examination to 24.375 after 2 months and was 23.75 after 6 months of treatment. The median scores of ADASCog fall from 42.125 at baseline to 38.0 after 2 months and to 35.0 after 6 months of treatment (Fig. 1).

The median score on DAD was $92.99 \%$ at baseline, $88.87 \%$ after 2 months and $88.85 \%$ after 6 months of treatment, showing a decrease in selfcare and in daily living activities. The median scores on CBS was 23.625 at baseline, 27.5 after 2 months of treatment and 32.75 after 6 months revealing a greater concern of the caregivers regarding their responsibility (Fig.1).

The disability caused by dementia increased on DAD although ADAS-Cog and MMSE scores improved; the concern of the caregivers, reflected by CBS scores, regarding their future and the future of the patient, increased as his disability grew.

\section{DISCUSSION}

We used for our periodic examination MMSE because, with all the limits mentioned before, it still is a valuable diagnostic and monitoring tool for neurocognitive impairment; ADAS-Cog is widely used in clinical trials for a more complex evaluation, although there are studies that suggest that ADAS-Cog is not the best tool for the examination of the patients with mild and moderate Alzheimer's disease, since certain items are not sensitive enough to detect the changes (18). In a meta-analysis, $\mathrm{K}$ Liu and colab. have demonstrated that Neuropsychological Battery Test (NBT) was more sensitive. Still, a lot of large trials use ADAS-Cog and MMSE, and U.S Food and Drug Administration has defined an improvement of four points on ADAS-Cog as a proof of clinical efficacy (19), while a decrease of three points in MMSE score is a sign of alteration 
TABLE 1. Demographic characteristics and associated diseases of the study group

\begin{tabular}{|l|c|c|c|c|c|c|c|c|}
\hline & Patient 1 & Patient 2 & Patient 3 & Patient 4 & Patient 5 & Patient 6 & Patient 7 & Patient 8 \\
\hline Sex & $\mathrm{F}$ & $\mathrm{F}$ & $\mathrm{M}$ & $\mathrm{M}$ & $\mathrm{F}$ & $\mathrm{M}$ & $\mathrm{M}$ & $\mathrm{F}$ \\
\hline Age (y) & 64 & 61 & 70 & 58 & 62 & 74 & 57 & 73 \\
\hline Education (y) & 10 & 16 & 8 & 16 & 10 & 16 & 16 & 12 \\
\hline Marital status & Single & Single & Married & Married & Married & Married & Married & Single \\
\hline Hypertension & Yes & No & Yes & Yes & Yes & No & Yes & Yes \\
\hline Dyslipidemia & Yes & Yes & Yes & Yes & Yes & No & Yes & Yes \\
\hline Diabetes & No & No & Yes & No & No & No & No & No \\
\hline Depression & No & Yes & No & No & No & No & No & No \\
\hline Antihypertensive drugs & Yes & No & Yes & Yes & Yes & No & Yes & Yes \\
\hline Statins & Yes & Yes & Yes & Yes & Yes & No & Yes & Yes \\
\hline Antidiabetic drugs & No & No & Yes & No & No & No & No & No \\
\hline Antidepressant drugs & No & Yes & No & No & Yes & No & No & No \\
\hline Antiplatelet drugs & No & No & Yes & Yes & No & No & Yes & Yes \\
\hline
\end{tabular}

TABLE 2. Results of the neurocognitive tests

\begin{tabular}{|l|c|c|c|c|c|c|c|c|}
\hline & Patient 1 & Patient 2 & Patient 3 & Patient 4 & Patient 5 & Patient 6 & Patient 7 & Patient 8 \\
\hline ADAS-Cog Baseline & 44 & 49 & 31 & 51 & 39 & 37 & 43 & 43 \\
\hline ADAS-Cog 2 m & 38 & 40 & 35 & 50 & 17 & 30 & 51 & 43 \\
\hline ADAS-Cog 6 m & 32 & 42 & 29 & 57 & 20 & 19 & 39 & 42 \\
\hline MMSE Baseline & 21 & 21 & 25 & 21 & 24 & 25 & 23 & 21 \\
\hline MMSE2 m & 22 & 22 & 28 & 21 & 24 & 30 & 27 & 22 \\
\hline MMSE 6 m & 21 & 20 & 29 & 20 & 28 & 25 & 25 & 22 \\
\hline CBS Baseline & 24 & 13 & 14 & 43 & 19 & 25 & 31 & 20 \\
\hline CBS 2 m & 56 & 8 & 30 & 25 & 35 & 14 & 38 & 14 \\
\hline CBS 6 m & 53 & 15 & 22 & 63 & 20 & 31 & 43 & 15 \\
\hline DAD Baseline & $20 / 40$ & $40 / 40$ & $36 / 36$ & $31 / 33$ & $40 / 40$ & $36 / 36$ & $37 / 37$ & $33 / 33$ \\
\hline DAD 2 m & $20 / 40$ & $40 / 40$ & $33 / 36$ & $31 / 33$ & $40 / 40$ & $33 / 36$ & $31 / 37$ & $33 / 33$ \\
\hline DAD 6 m & $34 / 40$ & $40 / 40$ & $35 / 36$ & $22 / 33$ & $40 / 40$ & $34 / 36$ & $25 / 37$ & $33 / 33$ \\
\hline
\end{tabular}

CB - Caregiver Burden Scale; DAD - Dementia Assessment Scale

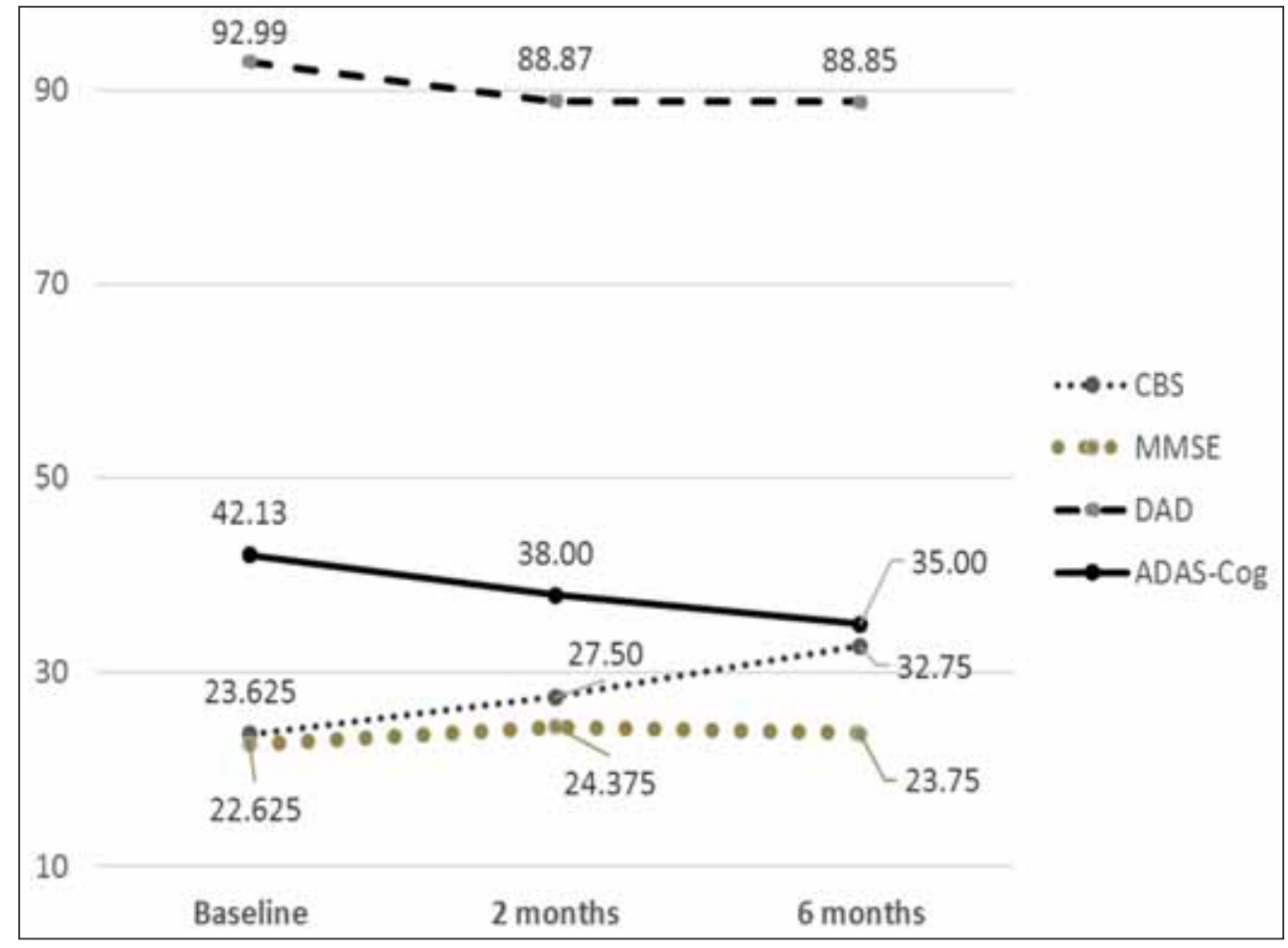

FIGURE 1. Median scores of ADAS-Cog decreased 7 points after 6 months of treatment, showing an improvement of cognitive function; the median functional disability score decreased by $4 \%$. The median MMSE score increased by 1.1 points after 6 months; the burden of the caregivers increased with 38\% after 6 months, as they understand and accept the course of the disease. CBS - Caregiver Burden Scale 
in cognitive ability (20). A discrepancy between cognitive and functional abilities was observed in clinical trials. In an open-label prospective study conducted on 734 patients with mild AD (MMSE between 20 and 26) treated with donepezil, rivastigmine or galantamine, patients were followed up to three years, on a bi-annual basis by Wattmo and colab. (21) using MMSE, ADAS- Cog, CIBIC and IADL scales. The authors concluded that after three years, patient's cognitive abilities and global performance were better preserved than their functional capacity. A higher mean dose of acethylcholinesterase inhibitors (regardless of drug agent), better preserved IADL abilities at baseline and a lower level of education were predictors of a slower cognitive decline, while younger age,lower educational level, living with a family member, better cognitive status and a higher ChEI dose were predictors of less functional deterioration.

ADAS-Cog score in our patients decreased after 2 and 6 months, with more than four points, confirming the good evolution of the cognitive function under treatment with galantamine. A similar pattern was obtained for MMSE scores, who increased 1.1 points, but the improvement in cognitive scores was not accompanied by a good evolution of the functional score on DAD Scale. In our group, half of the patients had a higher level of education (university degree), and all patients had at least 8 years of school. The number is too small to analyze the influence of education on individual scores, but it could be speculated that individuals with lower education have a reduced cognitive reserve, and are expected to perform worse in neurocognitive tests, so that they will develop earlier signs of disease, being diagnosed and treated earlier. The results published by Wattmo (21) are confirming by statistical methods, a well known clinical observation, that people with a higher cognitive reserve are diagnosed in later stages, because their level of knowledge allows them to mask the early signs of disease. It is though important to perform complex evaluation of the patients, including activities of daily living, since there may be a difference in the evolutive patterns of the cognitive and functional abilities, and as it was shown that an increased dose can lead to a slower deterioration in both, this could be a good instrument to adjust treatment in patients who receive acethylcholinesterase inhibitors. The impact on the caregiver has to be taken into account, because as the burden in- creases, this will lead on one side to health problems for the caregiver (depression, cardiovascular disease, even higher risk of death), but on the other side this can influence also the performance of the patient (confronted with a more hostile environment he will deteriorate faster)

Limits - the obvious limits of the studies with patients with Alzheimer's disease is the absence of control group (it is not considered ethical); the group of patients that we examined is small and possibly not representative.

\section{CONCLUSION}

Currently, the aim of the treatment of dementia is a better functional life, maintaining the capacity of self-care and an independent life of the patient. This raises the question if the instruments that we use in evaluating the response to the treatment are the right ones. ADAS-cog examination does not have a better prediction value after two months of treatment with galantamine compared with MMSE. Using the ADAS-cog score can be particularly efficient for the patients of whom MMSE score remain stable at six months of treatment, but it is not sufficient, since an improvement of ADAS-Cog and MMSE scores does not correlate with the functional scores on DAD. Functional scores on DAD do correlate with the scores on CBS; functional decline in social life affects also the life of their caregivers.

The treatment with Galantamine does improve the cognitive function, but not the social function; improvement of the social function might be obtained through other means such as occupational therapy. The health care system cannot provide an adequate care for these patients, so the informal costs are higher, rising the burden of the caregivers. The efficacy of the treatment could be proven only after the six month examination, while the test performed after two months of treatment did not have a predictive value for the further evolution of the patients taking Galantamine.

\section{Acknowledgement}

„This paper is partly supported by the Sectorial Operational Programme Human Resources Development (SOPHRD), financed by the European Social Fund and the Romanian Government under the contract number POSDRU 141531". 


\section{REFERENCES}

1. Hendrie H.C. Epidemiology of dementia and Alzheimer's disease. Am J Geriatr Psychiatry. 1998;6 (suppl 1)S3- S18

2. Bajenaru O.A., Tiu C., Antochi F., Roceanu A. Neurocognitive disorders in DSM 5 project - personal comments. J Neurol Sci. 2012 Nov 15; 322(1-2):17-9. doi: 10.1016/j.jns. 2012. 07.067. Epub 2012 Aug 19

3. Spencer R.J., Wendell C.R., Giggey P.P., Katzel L.I., Lefkowitz D.M., Siegel E.L., Waldstein S.R. Psychometric limitations of the mini-mental state examination among nondemented older adults: an evaluation of neurocognitive and magnetic resonance imaging correlates. Exp Aging Res 2013 May

4. Malek-Ahmadi M., Kora K., O’Connor K., Schofield S., Coon D., Nieri W. Longer self-reported sleep duration is associated with decreased performance on the montreal cognitive assessment in older adults. Aging ClinExp Res. 2015 Jun 11

5. Nakashima H., Umegaki H., Makino T., Kato K., Abe S., Suzuki Y., Kuzuya M. Neuroanatomical correlates of error types on the Clock Drawing Test in Alzheimer's disease patients. Geriatr Gerontol Int. 2015 Jul 14

6. Yang E., Farnum M., Lobanov V., Schultz T., Verbeeck R., Raghavan N., Samtani M.N., Novak G., Narayan V., DiBernardo. AQuantifying the pathophysiological timeline of Alzheimer's disease.; Alzheimer's Disease Neuroimaging Initiative. J Alzheimers Dis. 2011; 26(4)

7. Hashimoto M., Yatabe Y., Ishikawa T., Fukuhara R., Kaneda K., Honda K., Yuki S., Ogawa Y., Imamura T., Kazui H., Kamimura N., Shinagawa S., Mizukami K., Mori E., Ikeda M. Relationship between Dementia Severity and Behavioral and Psychological Symptoms of Dementia in Dementia with Lewy Bodies and Alzheimer's DiseasePatients. Dement GeriatrCogn Dis Extra. 2015 Jun 6

8. Isabelle Gélinas, Louise Gauthier, Maria McIntyre, Serge Gauthier, Development of a Functional Measure for Persons With Alzheimer's Disease: The Disability Assessment for Dementia American Journal of Occupational Therapy, September/October 1999, Vol. 53, 471-481. doi:10.5014/ajot.53.5.471 1999 September

9. O'Dwyer S.T., Moyle W., Taylor T., Creese J., Zimmer-Gembeck M.J. Homicidal ideation in family carers of people with dementia, Aging Ment Health. 2015 Jul 20.

10. Brown K.W., Coogle C.L., Wegelin J. A pilot randomized controlled trial of mindfulness-based stress reduction for caregivers of family members with dementia. Aging Ment Health. 2015 Jul 27:

11. Darbà J., Kaskens L. Relationship between patient dependence and direct medical-, social-, indirect-, and informal-care costs in Spain Clinicoecon Outcomes Res. 2015 Jul 2
12. Tay S.Y., Davison J., Jin N.C., Philip Y.L. Education and Executive Function Mediate Engagement in Advance Care Planning in Early Cognitive Impairment. J Am Med Dir Assoc. 2015 Jun 27

13. Liu-Seifert H., Siemers E., Sundell K., Price K., Han B., Selzler K., Aisen P., Cummings J., Raskin J., Mohs R. Cognitive and functional decline and their relationship in patients with mild Alzheimer's dementia. J Alzheimers Dis. 2015;43

14. Richard A. Hansen, Gerald Gartlehner, Aaron P. Webb, Laura C. Morgan, Charity G. Moore, Daniel E. JonasEfficacy and safety of donepezil, galantamine, and rivastigmine for the treatment of Alzheimer's disease: A systematic review and meta-analysis ClinInterv Aging. 2008 Jun; 3(2): 211-225.

15. Krista L. Lanctôt, Nathan Herrmann, Kenneth K. Yau, Lyla R. Khan, Barbara A. Liu, Maysoon M. Lou Lou, Thomas R. Einarson, Efficacy and safety of cholinesterase inhibitors in Alzheimer's disease: a meta-analysis CMAJ September 16, 2003 vol. 169 no. 6

16. Nhi-Ha Trinh, Jennifer Hoblyn, SubhanjoyMohanty, Kristine Yaffe, Efficacy of Cholinesterase Inhibitors in the Treatment of Neuropsychiatric Symptoms and Functional Impairment in Alzheimer Disease: A Meta-analysis JAMA. 2003; 289(2)

17. Journal of Alzheimer 's Disease, Feb 2013

18. J.D. Huntley, R.L. Gould, Psychometric evaluation of ADAS-Cog and NTB for measuring drug response. BMJ Open 2015

19. K. Liu, M. Smith, R.J. Howard, Do cognitive interventions improve general cognition in dementia? A meta-analisys and meta-regression, Acta Neurol Scand. 2014 Feb;129(2):114-22. doi: 10.1111/ane.12153. Epub 2013 Jun 13

20. Wilcock G.K., Lilienfeld S., Gaens E. Efficacy and safety of galantamine in patients with mild to moderate Alzheimer's disease: multicentrerandomised controlled trial: Galantamine International-1 study group. BMJ 2000, 321:1445-1449

21. Tariot P.N. Maintaining cognitive function in Alzheimer disease: how effective are current treatments? Alzheimer Dis Assoc Disord 2001, 15 (Suppl 1): S26 -S33

22. Carina Wattmo, Asa K. Wallin, Lennart Minthon, Progression of mild Alzheimer's Disease, knowledge and prediction required for future treatment strategies, Alzheimer Research\& Therapy, 2013

23. Asa K. Wallin, Carina Wattmo, Lennart Minthon, Galantamine treatment in Alzheimer's disease: response and long-term outcome in a routine clinical setting, Neuropsychiatric Disease and Treatment 2011:7 565-576 